\title{
Fixed point theorems for condensing multivalued mappings on a locally convex topological space
}

\section{E. Tarafdar and R. Výborný}

\begin{abstract}
A general definition for a measure of nonprecompactness for bounded subsets of a locally convex linear topological space is given. Fixed point theorems for condensing multivalued mappings have been proved. These fixed point theorems are further generalizations of Kakutani's fixed point theorems.
\end{abstract}

\section{Introduction}

Using the concept of condensing mapping Sadovskii [11] and Lifšic and SadovskiT [9] have obtained respectively the generalizations of Schauder [12] and Tychonoff [14] fixed point theorems. Daneś [2] has obtained the generalization of Kakutani's fixed point theorem [7] by using the concept of multivalued condensing mapping. Reinermann [10] has also used condensing mapping defined in terms of a measure of noncompactness (nonprecompactness) of bounded sets to obtain generalizations of Schauder Theorem [12]. Using the multivalued condensing mapping defined in terms of a measure of precompactness Himmelberg, Porter and van Vleck [6] have proved a fixed point theorem which includes the fixed point theorems of Sadovski [11], Tychonoff [14], Glicksberg [5], Fan [4] and a part of a theorem of Browder [1].

The aim of this note is to obtain a fixed point theorem which will contain the above fixed point theorems of $[2,4,5,6,7,9,10,11,12$, 14].

Received 8 November 1974 . 
In $\$ 2$ we have introduced a general definition of a measure of nonprecompactness of bounded sets in a locally convex linear topological space. In $\S 3$ we have given various definitions of condensing multivalued mappings and have unified them in a single definition. In $\S 4$ we have proved our main fixed point theorem and also obtained corollaries and a theorem which are similar but more general than the corresponding corollaries and theorem of $[6]$.

We follow the notation and terminology as in [6]. A multivalued mapping $F: X \rightarrow Y$ is a mapping which assigns to each point $x \in X$ a nonempty set $F(x)$ of $Y, F$ is a subset of $X \times Y$ whose domain is $F^{-1}(Y)=X$. The set $\{(x, y): x \in X$ and $y \in F(x)\}$ is called the graph of $F$ or simply $F$. For $\Omega \subset X$, a multivalued mapping $G: \Omega \rightarrow Y$ having the property that $G(x) \subset F(x)$ for each $x \in \Omega$ is called a submultivalued mapping of $F$. For $\Omega \subset X, F(\Omega)=U F(x)$.

A point $x \in X$ is a fixed point of a multivalued mapping $F: X \rightarrow X$ if $x \in F(x)$. It is obvious that a fixed point of a submultivalued mapping of $F$ is also a fixed point of $F$.

A multivalued mapping $F: X \rightarrow Y$ of a topological space $X$ into a topological space $Y$ is called upper semicontinuous if for each closed subset $A$ of $Y, F^{-1}(A)$ is closed. $F$ has closed graph if $Y$ is regular and $F$ is upper semicontinuous and has closed values (see [8], p. 175). A multivalued mapping $F: X \rightarrow Y$ is lower semicontinuous if for each open subset $A$ of $Y, F^{-1}(A)$ is open.

In the sequel (E, $\tau)$ will always denote a locally convex linear topological space, and $\left[p_{\alpha}: \alpha \in I\right]$ will denote the family of seminorms which generates the topology $\tau$. Any topological concept, such as closedness, precompactness, compactness, boundedness, and so on, will be understood as 'with respect to the topology $\tau$ '. In all other cases, that is, when a topological concept is not meant with respect to $\tau$, the corresponding topology will precede the concept; for example, $p_{\alpha}$-precompact to mean that certain subset is precompact with respect to $p_{\alpha}$-topology 


\section{Measure of precompactness and nonprecompactness}

We denote by $C$ the class of all bounded subsets of $(E, \tau)$.

DEFINITION 2.1. $\mu=\left[\mu_{\alpha}: \alpha \in I\right]$ will be said to define a measure of precompactness on $C$, where for each $\alpha \in I, \mu_{\alpha}$ is a set (interval) valued mapping of $C$ into $R^{+}$, the set of non-negative real numbers, having properties:

(i) $\mu_{\alpha}(\Omega)=[a, \infty)$ or $(a, \infty), a \geq 0$ for each $\Omega \in C$;

(ii) $\Omega_{1} \subset \Omega_{2} \in C$ implies $\mu_{\alpha}\left(\Omega_{1}\right) \supset \mu_{\alpha}\left(\Omega_{2}\right)$ for every $\alpha \in I$;

(iii) $\mu_{\alpha}(\Omega)=\mu_{\alpha}(\cos \Omega)$ for each $\Omega \in C$ where co $\Omega$ stands for the convex hull of $\Omega$;

(iv) $\mu_{\alpha}\left(\Omega_{1} \cup \Omega_{2}\right)=\mu_{\alpha}\left(\Omega_{1}\right) \cap \mu_{\alpha}\left(\Omega_{2}\right)$ for $\Omega_{1}, \Omega_{2} \in \mathrm{C}$;

(v) $\mu_{\alpha}(\Omega)=R^{+}$if $\Omega$ is precompact and $\Omega$ is precompact if $\mu_{\alpha}(\Omega) \supset(0, \infty)$, for each $\alpha \in I$.

For $\Omega \in C, \hat{\mu}(\Omega)=\left[\hat{\mu}_{\alpha}(\Omega): \alpha \in I\right]$ where $\hat{\mu}_{\alpha}(\Omega)=\inf \mu_{\alpha}(\Omega)$ may then be regarded as a measure of nonprecompactness of $\Omega$. Thus all the entries in the parenthesis of $\hat{\mu}(\Omega)$ are zeros if and only if $\Omega$ is precompact.

EXAMPLE 2.1 (Kuratowski). For each $\Omega \in C$, we define $\lambda(\Omega)=\left[\lambda_{\alpha}(\Omega): \alpha \in I\right]$ where $\lambda_{\alpha}(\Omega)=\{\varepsilon>0: \Omega$ can be covered by a finite number of sets of

$$
\left.p_{\alpha} \text {-diameter } \leq \varepsilon\right\} \text {. }
$$

Then $\lambda$ is indeed a measure of precompactness on $C$.

(i) $\lambda_{\alpha}(\Omega)=\left[\hat{\lambda}_{\alpha}(\Omega), \infty\right)$ or $\left(\hat{\lambda}_{\alpha}(\Omega), \infty\right) \cdot$ (ii), (iv), and (v) follow easily. For proof of (iii) we refer to Darbo [3] (the proof given by Darbo for a normed space applies also for a seminormed space).

EXAMPLE 2.2. Let $U_{\alpha}=\left\{x \in E: p_{\alpha}(x) \leq 1\right\}$.

For each $\Omega \in \mathcal{C}$, we define $\gamma(\Omega)=\left[\gamma_{\alpha}(\Omega): \alpha \in I\right]$ where 
$\gamma_{\alpha}(\Omega)=\left\{\varepsilon>0:\right.$ there exists a $p_{\alpha}$-precompact subset $S$ with

$$
\left.S+\varepsilon U_{\alpha} \supset \Omega\right\}
$$

(i) As before we take $a=\hat{p}_{\alpha}(\Omega)$. The proof of (ii) and (iv) is trivial.

(iii) In view of (ii) it suffices to show that $\gamma_{\alpha}(\Omega) \subset \gamma_{\alpha}(\cos )$. Let $t \in \gamma_{\alpha}(\Omega)$. Then there exists a $p_{\alpha}$-precompact subset $S$ such that $s+t U_{\alpha} \supset \Omega$. Since $\cos +t U_{\alpha} \supset \Omega$ and $\cos +t U_{\alpha}$ is convex, $\cos +t U_{\alpha} \supset \cos$. Noting that $\cos$ is $p_{\alpha}$-precompact, we conclude that $t \in \gamma_{\alpha}(\cos \Omega)$

(v) Let $\Omega$ be $\tau$-precompact. Then $\Omega$ is $p_{\alpha}$-precompact for each $\alpha \in I$. Since $\Omega+t U_{\alpha} \supset \Omega$ for all $t \geq 0$ and $\alpha \in I, \mu_{\alpha}(\Omega)=R^{+}$for all $\alpha \in I$.

Next, let $\alpha \in I$ be arbitrary and $\mu_{\alpha}(\Omega) \supset(0, \infty)$.

Let $r>0$ be any real number. Since $\frac{r}{2} \in \mu_{\alpha}(\Omega)$, there exists a $p_{\alpha}$-precompact set $S$ such that $S+\frac{r}{2} U_{\alpha} \supset \Omega$. Since $S$ is $p_{\alpha}$-compact, there exists a finite set $F$ such that $F+\frac{r}{2} U_{\alpha} \supset S$. Now $F+r U_{\alpha} \supset S+\frac{r}{2} U_{\alpha} \supset \Omega$. Thus $\Omega$ is $p_{\alpha}$-precompact. Since $\alpha$ is arbitrary, $\Omega$ is $\tau$-precompact.

EXAMPLE 2.3. Let $(E, \tau),\left[p_{\alpha}: \alpha \in I\right]$ and $C$ be as before. For $\Omega \in C$, we define $\nu(\Omega)=\left[\nu_{\alpha}(\Omega): \alpha \in \Omega\right]$ where $\nu_{\alpha}(\Omega)=\{\varepsilon>0:$ there exists a precompact set $S$ such that $\left.S+\varepsilon U_{\alpha} \supset \Omega\right\}$

The proof that $v$ is a measure of nonprecompactness on $C$ is similar to that of Example 2.2. We note that for each $\Omega \in C$, $\nu_{\alpha}(\Omega) \subset \gamma_{\alpha}(\Omega)$ for each $\alpha \in I$. 


\section{Condensing mappings}

Himmelberg, Porter and van Vleck [6] have defined a measure of precompactness for any subset of $(E, \tau)$ in the following way.

Let $B$ be a base of convex neighbourhoods of 0 . Then for $\Omega \subset E$, $Q(\Omega)$, the measure of precompactness of $\Omega$, is defined to be the collection of all $B \in B$ such that $S+B \supset \Omega$ for some precompact subset $S$ of $E$. With this notion of measure of precompactness they have introducted a definition of condensing mapping.

Let $X$ be a nonempty subset of a locally convex linear topological space $(E, \tau)$. Let $\left[p_{\alpha}: \alpha \in I\right]$ and $C$ be as before. Let $F: X \rightarrow X$ be a multivalued mapping.

DEFINITION 3.1. $F$ is condensing with respect to $Q$ if for each $\tau$-bounded but not $\tau$-precompact set $\Omega \subset X$ with $F(\Omega) \subset \Omega$ we have $Q(F(\Omega)) \supsetneqq Q(\Omega)$.

DEFINITION 3.2. $F$ is condensing with respect to $\mu$ if for each bounded but not precompact set $\Omega \subset X$ with $F(\Omega) \subset \Omega$, there exists a $\alpha \in I$ such that $\hat{\mu}_{\alpha}(F(\Omega))<\hat{\mu}_{\alpha}(\Omega)$ where $\mu=\left[\mu_{\alpha}: \alpha \in I\right]$ is a measure of precompactness on $\mathrm{C}$.

DEFINITION 3.3. $F$ is condensing with respect to $\mu$ if for each bounded but not precornpact set $\Omega \subset X$ with $F(\Omega) \subset \Omega$, there exists $\alpha \in I$ such that $\mu_{\alpha}(F(\Omega)) \supsetneqq \mu_{\alpha}(\Omega)$.

DEFINITION 3.4. $F$ is condensing if for each $\Omega \subset X$ with $F(\Omega) \subset \Omega$,

(a) the condition that $\Omega-\operatorname{clcoF}(\Omega)$ is compact implies the compactness of $\mathrm{cl} \Omega$; or

(b) the condition that $\Omega-\operatorname{coF}(\Omega)$ is empty or single point implies the compactness of $c l \Omega$.

DEFINITION 3.5. $F$ is condensing if for each $\Omega \subset X$ with $F(\Omega) \subset \Omega$, the condition that $\Omega-\operatorname{co} F(\Omega)$ is empty or single point implies that $\Omega$ is precompact.

Definition 3.1 is due to Himmelberg, Porter and van Vleck [6]. For a single valued mapping, Definition 3.2 has been used by Reinermann [10] and Stallbohm [13] with $\mu=\lambda$, and Definition 3.4 is due to Lifšic and 
Sadovskit [9]. Definition 3.5 is a slight variant of the one given by Daněs [2].

(A) It is easy to see that Definition 3.2 implies Definition 3.3 for each measure $\mu$.

(B) Definition 3.1 implies Definition 3.3.for suitable measure $\mu$. Let Definition 3.1 hold. We index the base $B$ by $B=\left|B_{\alpha}: \alpha \in I\right|$. Let $p_{\alpha}$ be the Minkowski functional on $B_{\alpha}$. Let $U_{\alpha}=\left\{x \in E: p_{\alpha}(x) \leq 1\right\}$. Clearly $B_{\alpha}=U_{\alpha}$. We now consider the measure $\nu$ as defined in Example 2.3. We now show that Definition 3.3 holds with respect to $\nu$. Let $\Omega$ be any bounded but not precompact subset of $X$ with $F(\Omega) \subset \Omega$. Then we have $Q(F(\Omega)\} \supsetneqq Q(\Omega)$; that is, there exists a $B_{\alpha} \in B$ such that $B_{\alpha} \in Q(F(\Omega))$ but $B_{\alpha} \vDash Q(\Omega)$. Hence it follows that $1 \in \nu_{\alpha}(F(\Omega))$ but $1 \notin v_{\alpha}(\Omega)$. Also since $F(\Omega) \subset \Omega$, it follows from (ii) of Definition 2.1 that $\nu_{\alpha}(F(\Omega)) \supsetneqq \nu_{\alpha}(\Omega)$.

(C) Definition 3.3 with each measure $\mu$ implies Definition 3.5 if $F$ has bounded range. Let Definition 3.3 hold with a measure $\mu$. Let $\Omega \subset X, F(\Omega) \subset \Omega$, and $\Omega-\operatorname{coF}(\Omega)=Z$ where $Z=\emptyset$ or a single point. Obviously $\mu_{\alpha}(Z)=R^{+}$for each $\alpha \in I$.

Since $\Omega \subset Z \cup \operatorname{co} F(\Omega)$, it follows that $\Omega$ is bounded and we have for each $\alpha \in I, \mu_{\alpha}(\Omega) \supset \mu_{\alpha}(Z u \operatorname{co} F(\Omega))$ by (ii) of Definition $2 . I$ equal to $\mu_{\alpha}(Z) \cap \mu_{\alpha}(F(\Omega))$ by (iv) and (iii) of Definition 2.1. Again since $\Omega \supset Z \cup F(\Omega)$, we have for each $\alpha \in I, \mu_{\alpha}(\Omega) \subset \mu_{\alpha}(Z) \cap \mu_{\alpha}(F(\Omega))$ by (ii) and (iv) of Definition 2.1. Thus for each $\alpha \in I$, $\mu_{\alpha}(\Omega)=\mu_{\alpha}(Z) \cap \mu_{\alpha}(F(\Omega))$. From this and the fact that $\mu_{\alpha}(z)=R^{+}$for each $\alpha \in I$, it follows that $\mu_{\alpha}(\Omega)=\mu_{\alpha}(F(\Omega))$ for each $\alpha \in I$, which in view of Definition 3.3 implies that $\Omega$ is precompact.

(D) Obviously Definition 3.4 implies Definition 3.5.

\section{Fixed point theoreins}

The proof of the following lemma can be found in [6]. 
LEMMA 4.1. Let $X$ be a topological space. Let $F: X+X$ be a miltivalued mapping with closed graph. If there exists a nonempty subset $A$ of $X$ such that $F(A) \subset A$ and $C \perp A$ is compact, then there exists a nonempty, closed and compact subset $K$ of $X$ such that $K \subset F(K)$.

THEOREM 4.1. Let $X$ be a nonempty complete convex subset of a Hausdorff locally convex linear topological space $E$. Let $F: X \rightarrow X$ be a condensing multivalued mapping in the sense of Definition 3.5 with convex values and closed graph. Then $F$ has a fixed point.

Proof. Unlike [11], [9], and [6], we will not use ordinals. Let $x \in X$. Set $A=\{x\} \cup\left\{\bigcup_{n=1}^{\infty} F^{n}(x)\right\}$. Then clearly $F(A) \subset A$ and $A-\operatorname{coF}(A) \subset\{x\}$. Since $F$ is condensing, $A$ is precompact. Also $c I A \subset X$ and $c I A$ is compact as $X$ is complete. Hence by Lemma 4.I, there exists a nonempty compact subset $K$ of $X$ such that $F(K) \supset K$.

Let $S=\{Y \subset X: K \subset Y, F(Y) \subset Y$ and $Y$ is convex $\} . S$ is nonempty as $X \in S . S$ is a partially ordered set with respect to the reiation $\leq$ where $Y_{1} \leq Y_{2}$ if and only if $Y_{1} \supset Y_{2}$ with $Y_{1}, Y_{2} \in S$.

We first prove that every chain in $S$ has an upper bound in $S$. Let $T$ be a chain in $S$. Then $Z=\bigcap Y$ is an upper bound. Clearly $Y \in T$

$Z \subset X, K \subset Z, F(Z) \subset Z$, and $Z$ is convex. Hence $Z \in S$. Thus by Zorn's Lemma there is a maximal element $z_{0} \in S$.

We next prove that for each $Y \in S, \operatorname{coF}(Y) \in S$.

(a) $\operatorname{coF}(Y) \subset X$ as $F(Y) \subset Y \subset X$ and $X$ is convex.

(b) $K \subset \operatorname{coF}(Y)$.

Since $K \subset Y$ and $K \subset F(K)$, we have $K \subset F(K) \subset F(Y)$. Hence $K \subset \operatorname{coF}(Y)$.

(c) $F(\operatorname{coF}(Y)) \subset \operatorname{coF}(Y)$.

Since $F(Y) \subset Y$ and $Y$ is convex, $\operatorname{co} F(Y) \subset Y$. Hence $F(\operatorname{co} F(Y)) \subset E(Y) \subset \operatorname{co} F(Y)$.

(d) $\operatorname{coF}(Y)$ is convex.

Now since for each $Y \in S, F(Y) \subset Y$ and $Y$ is convex, we have 
$\operatorname{coF}(Y) \subset Y$. Thus $Y \leq \operatorname{coF}(Y)$ for each $Y \in S$. In particular $Z_{0} \leq \operatorname{coF}\left(Z_{0}\right)$. But since $Z_{0}$ is a maximal element in $S$, it follows that $z_{0}=\operatorname{coF}\left(z_{0}\right) ;$ that is, $z_{0}-\operatorname{coF}\left(z_{0}\right)=\emptyset$. Hence by condensing of $F$, $Z_{0}$ is precompact. Therefore, $c l Z_{0} \subset X$ and $c l Z_{0}$ is compact. The rest of the argument is as given in [6]. Let $G=F \cap\left(\operatorname{clz} x_{0} \times c l Z_{0}\right)$. Then $G$ is closed and compact subset of $X \times X$. Also $G^{-1}\left(\mathrm{cIZ}_{0}\right)$ is a closed subset of $c 1 Z_{0}$ containing $Z_{0}$. Thus domain $G=G^{-1}\left(\operatorname{cl} Z_{0}\right)=c l Z_{0}$. Hence $G$ is a multivalued mapping of $c l Z_{0}$ into $c l Z_{0}$, with convex values ard compact graph. ( $G$ is also upper semicontinuous.) Hence by the theorem of Glicksberg [5] or of Fan [4], $G$ has a fixed point in $c 1 Z_{0}$. This fixed point is also a fixed point of $F$.

REMARKS 4.1. The same remark as given in ([6], p. 637) applies in the present situation; that is, the above theorem remains true for non Hausdorff (nonseparated) $E$ if the further assumption that $X$ is closed is assumed. For details see [6] as quoted above.

REMARK 4.2. If $F$ is assumed to be condensing with respect to Definition 3.4, then the above theorem remains true with the completeness condition on $X$ replaced by the condition that $X$ is closed. The same proof applies, because in this case $c l A$ and $c l Z_{0}$ appeared in the proof would be compact directly due to the condensing of $F$. By Remark 4.I we can then remove the Hausdorfe condition on $E$ as the condition that $X$ is closed is already assumed. The resulting version of the theorem will include fixed point theorems of Lifšic and Sadovskił [9].

COROLLARY 4.1. Let $X$ be a nonempty complete convex subset of a Hausdorff locally convex linear topological space $E$. Let $F: X \rightarrow X$ be a multivalued mapping with convex values, closed graph and bounded range. If $F$ is condensing in the sense of Definition 3.3, then $F$ has a fixed point.

Proof. This follows from Theorem 4.1 and (C) of $\$ 3$.

REMARK 4.3. In view of $(B), \S 3$, it follows that the fixed point theorem of Himmelberg, Porter and van Vleck ([6], Theorem 1 ) is a special 
case of our Corollary 4.1.

The following theorem includes the corresponding theorem of ( $[6]$, Theorem 3).

THEOREM 4.2. Let $X$ be a nonempty complete convex subset of a locally convex linear topological space $E$. Let $F: X \rightarrow X$ be a lower semicontinuous multivalued mapping with closed convex values. Then $F$ has a fixed point if either of the following conditions hold:

(a) $X$ is compact and metrizable;

(b) the subspace uniformity on $X$ is metrizable and $F$ is condensing in the sense of Definition 3.5 .

Proof. (a) Same proof as in [6] applies.

(b) We proceed as in the proof of Theorem 4.1 until the set $z_{0}$ with $\operatorname{coF}\left(z_{0}\right)=z_{0}$ is obtained. By Corollary 2a, p. 176 of [8], $F\left(\operatorname{cl} z_{0}\right) \subset \operatorname{clF}\left(z_{0}\right) \subset \operatorname{cl} Z_{0}$. We then apply case $(a)$ to $F \cap\left(c 1 Z_{0} \times c l z_{0}\right): c l Z_{0} \rightarrow c l z_{0}$. For details see [6].

\section{References}

[1] Felix E. Browder, "On a generalization of the Schauder fixed point theorem", Duke Math. J. 26 (1959), 291-303.

[2] Josef Daneš, "Some fixed point theorems", Comment. Math. Univ. Carolinae 9 (1968), 223-235.

[3] Gabriele Darbo, "Punti uniti in trasformazioni a codominio non compatto", Rend. Sem. Mat. Univ. Padova 24 (1955), 84-92.

[4] Ky Fan, "Fixed-point and minimax theorems in locally convex topological linear spaces", Proc. Nat. Acad. Sci. U.S.A. 38 (1952), 12I-126.

[5] I.L. Glicksberg, "A further generalization of the Kakutani fixed point theorem, with application to Nash equilibrium points", Proc. Amer. Math. Soc. 3 (1952), 170-174. 
[6] C.J. Himmelberg, J.R. Porter and F.S. van Vleck, "Fixed point theorems for condensing multifunctions", Proc. Amer. Math. Soc. 23 (1969), 635-641.

[7] Shizuo Kakutani, "A generalization of Brouwer's fixed point theorem", Duke Math. J. 8 (1941), 457-459.

[8] K. Kuratowski, Topology, Vol. I (New edition, revised and augmented. Translated by J. Jaworowski. Academic Press, New York, London; Państwowe Wydawnictwo Naukowe, Warsaw; 1966).

[9] E.A. Лнфшнц, Б.H. Садовсний [E.A. Lif̌́ic, B.N. Sadovskiǐ], "Teopema o неподвинной точне для обобщенно уплотняющи операторов" [A fixed-point theorem for generalized condensing operators], Dokl. Akad. Nauk SSSR 183 (1968), 278-279; English translation: Soviet Math. Dokz. 9 (1968), 1370-1372.

[10] Jochen Reinermann, "Fixpunktsätze vom Krasnoselski-Typ", Math. Z. 119 (1971), 339-344.

[11] B.N. SadovskiY, "A fixed-point principle", Functional AnaZ. App I. I (1967), 151-153.

[12] J. Schauder, "Der Fixpunktsatz in Funktionalräumen", Studia Math. 2 (1930), 171-180.

[13] Volker Stallbohm, "Fixpunkte nichtexpansiver Abbildungen, Fixpunkte kondensierender Abbildungen, Fredholm'sche Sätze linearer kondensierender Abbildungen", (Rheinisch-Westfälische Technische Hochschule Aachen, Dr. Nat. Dissertation, 1973).

[14] A. Tychonoff, "Ein Fixpunktsatz", Math. Ann. 111 (1935), 767-776.

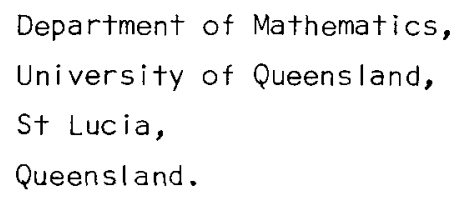

